\title{
Open Banking und standardisierte Schnittstellen auf dem Finanzplatz Schweiz
}

\author{
Marco Birkhofer und Sandro Bächli
}

\section{Zusammenfassung}

Die Studie untersucht die Auswirkungen von Open Banking und standardisierten Schnittstellen auf die Schweizer Bankenindustrie. Ziel war es, den Stand von Open Banking in der Schweiz, die Chancen und Risiken des Ansatzes sowie den Einfluss auf die Geschäftsmodelle zu untersuchen und daraus Handlungsempfehlungen für die Praxis abzuleiten. Es wurde aufgezeigt, dass Open Banking in der Schweiz noch in den Anfängen steckt und gegenüber den Vorreitern aus Großbritannien und der Europäischen Union Nachholbedarf besteht. Open Banking bietet die Chance, mit innovativen Services das bestehende Angebot zu erweitern und so die Customer Experience auf ein neues Level zu heben. Weitere Chancen sind Partnerschaften mit FinTechs und der Aufbau von Ökosystemen. Die etablierten, geschlossenen Geschäftsmodelle müssen hierzu aufgebrochen werden und der Trend deutet auf digitale Ökosysteme mit klarem Kundenfokus hin.

\subsection{Einleitung}

Die Schweiz gilt seit langer Zeit als weltweit führendes Land im Bankenwesen. Im Inland ermöglichen Banken den täglichen Geldfluss und übernehmen das Risikomanagement, wobei insbesondere Markt- und Kreditrisiken traditionell unterschieden werden. Private

M. Birkhofer

Biel, Schweiz

S. Bächli $(\bowtie)$

Berner Fachhochschule Wirtschaft, Bern, Schweiz

E-Mail: sandro.baechli@bfh.ch 
Haushalte, Unternehmen und nicht zuletzt der Staat sind auf vielfältige Weise auf diese Funktionen der Banken angewiesen. Der Bankensektor ist aber auch für die internationale Wettbewerbsfähigkeit der Schweiz einer der wichtigsten Treiber. Angesichts der technologischen, demografischen, sozialen und politischen Veränderungen durchläuft die Branche zurzeit einen fundamentalen Wandel (PWC 2018, S. 5). Die Digitalisierung ist neben den anderen Faktoren der signifikanteste Trend mit großen Auswirkungen auf Banken und deren Kunden. ${ }^{1}$ Die Branche steht daher vor einer Reihe Herausforderungen. Viele der etablierten Institute bekunden dennoch Mühe, ihre IT-Plattformen bzw. ihre IT-Architektur zu modernisieren und digital so auszurichten, dass sie ihre Kosten senken und die Kundeninteraktion verändern können (Deloitte 2016, S. 3). Im Angesicht dieser Herausforderungen bietet Open Banking die Möglichkeit, den Bankensektor zu öffnen, Innovationen zu fördern und die Interaktion und das Erlebnis von Finanzdienstleistungen radikal zu verbessern (Accenture 2018, S. 4).

Hier knüpft die Forschungsarbeit an. In der Europäischen Union (EU) ist mit der im Januar 2018 in Kraft getretenen Zahlungsdiensterichtlinie (PSD2) große Bewegung ins Bankengeschäft gekommen. Die Banken verlieren - durch gesetzlichen Zwang - ihr bisheriges Monopol auf Kundendaten. Denn auf Wunsch der Kunden müssen die Banken diese an Drittanbieter weitergeben. Die Einführung ermöglicht nicht nur FinTechUnternehmen mit neuen Angeboten in das bisherige Geschäft der Banken vorzustoßen, sondern auch den großen Technologieunternehmen wie Google, Facebook, Amazon oder Apple. Die Schweizer Banken sehen die Einführung einer ähnlichen Richtlinie kritisch, und auch die Schweizer Bankiervereinigung lehnt eine Regulierung ab (Schweizerische Bankiervereinigung 2017). Auch ohne gesetzlichen Zwang wird Open Banking die Überlegungen der Schweizer Banken und FinTechs künftig zunehmend prägen. Es ist eine Frage der Zeit, bis Kunden die mit Open Banking verbundenen Möglichkeiten fordern. Jene Banken, die das Thema proaktiv angehen, dürften in Zukunft erfolgreicher sein (NZZ 2018).

\subsection{Digitale Transformation und Open Banking}

Die Literatur unterscheidet zwischen „Digitalisierung“ und „Digitaler Transformation“. Letztere wird durch die Digitalisierung ermöglicht (Peter 2017, S. 9) und kann einerseits aus einem technischen Blickwinkel, anderseits aus einem gesellschaftlichen Gesichtspunkt betrachtet werden (Matt et al. 2015, S. 340). Aus technischer Sicht ist der Begriff eng mit dem vorgelagerten Schritt „Digitization“ verbunden. Dieser bezeichnet den Prozess der Umwandlung von analogen in digitale Daten. „Liegen die Daten in digitaler Form vor, so können diese über unterschiedliche Medien transportiert sowie grundsätzlich auf unterschiedlichen Endgeräten präsentiert werden“ (Matt et al. 2015, S. 339). Aus gesell-

\footnotetext{
${ }^{1}$ Im Folgenden wird aus Gründen der besseren Lesbarkeit ausschließlich die männliche Form benutzt. Es können dabei sowohl männliche als auch weibliche Personen gemeint sein.
} 
schaftlicher Sicht beruht die Digitalisierung auf dem Begriff der „Informatisierung“ (Nora und Minc 1979). Dieser fokussiert auf die „Durchdringung aller Lebensbereiche der Gesellschaft mit IT und damit verbundenen Möglichkeiten bzw. Veränderung" (Alt und Puschmann 2016, S. 22). Der Fokus dieser Forschung liegt auf der zweiten Perspektive, welche sich insbesondere mit der „Transformierung der Geschäftsmodelle, Wertschöpfungsketten, Geschäftsprozesse, IT- bzw. Analytics-Systeme und/oder von der Organisation selbst" (Fasel und Meier 2016, S. 321) beschäftigt. Die Einteilung der Zeiträume erfolgte hinsichtlich des Einsatzes von Informationstechnologien (Alt und Puschmann 2016, S. 36-40). Die Digitalisierung der Banken befindet sich folglich gemäß Abb. 6.1 in der vierten Phase. Gekennzeichnet wird die vierte Phase gemäß den Autoren durch eine, auf der internen Integration aufbauende, weitergehend betriebliche Integration. Die in der dritten Phase etablierten Kernbankensysteme, werden nun verstärkt modular konzeptioniert. Es wird vermehrt auf serviceorientierte Architekturen gesetzt, die eine Herauslösung einzelner funktionaler Bereiche (bzw. Services) ermöglichen, welche anschließend an Dienstleister vergeben werden können (Alt und Puschmann 2016, S. 39). In diese vierte Phase fällt auch die FinTech-Evolution mit neuen innovativen Lösungen zur Verbesserung der Kundeninteraktion. Dabei beteiligen sich Banken und Start-up-Unternehmen (Ornau 2017, S. 51). In der fünften Phase könnte ein Wechsel von einem bisher produktorientierten Paradigma in eine kundenzentrierte Perspektive stattfinden. Elektronische Plattformen ersetzen die bilateralen Beziehungen zwischen Bank und Kunden bzw. Dienstleistern. Es entstehen multilaterale Beziehungen, die Kunden und Dienstleistern gleichermaßen ermöglichen, über eine Schnittstelle (zum Marktplatz) eine Integration herstellen zu können (Alt und Puschmann 2016, S. 39). Eine hybride Kundeninteraktion entsteht, da ein Austausch von bankrelevanten Informationen über mehrere Kanäle (Mobil, Web, Filiale, Nicht-Bank etc.) möglich ist (Alt und Puschmann 2016, S. 40).

Die „digitale Transformation“ bezeichnet den Wandel, welcher mit der Digitalisierung und der Weiterentwicklung der Technologie in Verbindung steht (Peter 2017). Dies hat Folgen für die Wirtschaft wie auch für die Gesellschaft, da es unser Alltagsleben verändert (Pousttchi 2017). Die Digitale Transformation wird auch als die vierte industrielle Revolution bezeichnet (Peter 2017). Das Hauptaugenmerk der Digitalen Transformation liegt auf der Veränderung von Unternehmen im Zeitalter der Digitalisierung. Eine weitere Begriffsdefinition folgt vom MIT Center for Digital Business: „The use of new digital technologies (social media, mobile, analytics or embedded devices) to enable major business improvements (such as enhancing customer experience, streamlining operations or creating new business models)" (Fitzgerald et al. 2013).

Den Fokus nur auf die Anwendung neuer Technologien zur Weiterentwicklung der Geschäftsmodelle und der Angebote zu legen, erscheint nicht genügend zu sein. Dies, obwohl der schnell fortschreitende Wandel insbesondere Unternehmen vor viele Herausforderung stellt und diese möglichst agil sein müssen. Aus der Literatur lässt sich ableiten, dass neben neuen Anwendungen auch weitere Faktoren die Transformation beeinflussen. Die Interaktion zwischen den Kunden und den Unternehmen scheint dabei der Haupttreiber zu sein. Ein Hauptaugenmerk gilt hier der Art und Weise, wie Menschen mit den 

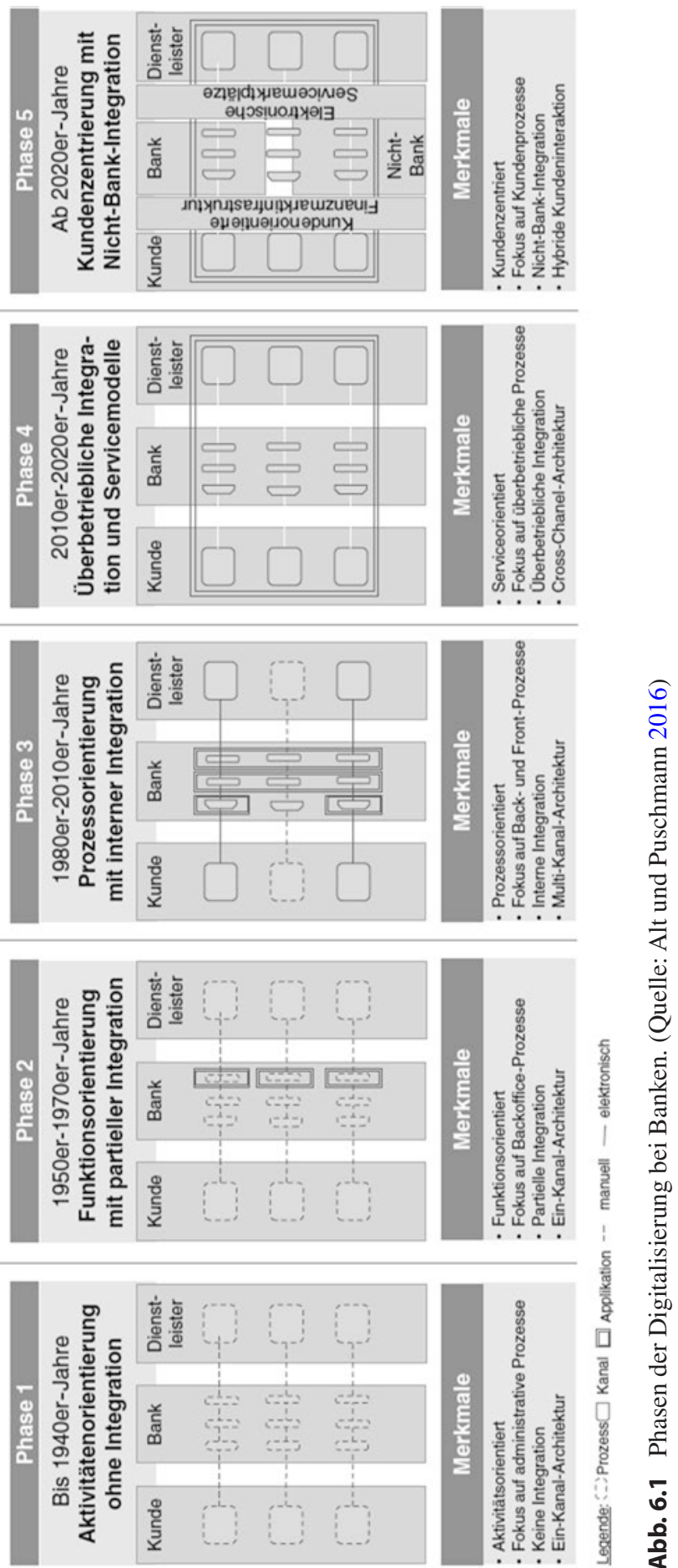

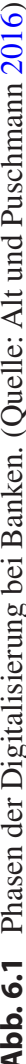


digitalen Möglichkeiten ihren Alltag gestalten. Es entstehen dabei neue Anforderungen und Bedürfnisse der Kunden. Die Möglichkeit, mit Open Banking die Customer Experience zu verbessern, ist stark von der Akzeptanz der Kunden abhängig. Dieser Aspekt steht jedoch nicht im Zentrum der Forschungsarbeit. Der Fokus der Untersuchung liegt auf den Unternehmen bzw. Banken. Eine Einordnung der digitalen Transformation und ihrer Folgen für Unternehmen ist demnach für die Forschungsarbeit wichtig. Mit der Perspektive auf die Unternehmen wird auch der Begriff „Digital Business Transformation“ verwendet. Die folgende Definition des Global Centers for Business Transformation verdeutlicht dies: „Digital Business Transformation is Organizational Change through the use of Digital Technologies and Business Models to improve Performance“ (Wade 2015). In diesem Kontext findet eine Unterscheidung zwischen den drei Wirkungsdimensionen Leistungserstellung, Leistungsangebot und Kundeninteraktion statt (Pousttchi 2017).

Open Banking hat das Potenzial, Auswirkungen auf alle drei Bereiche zu haben. Unter Open Banking versteht sich sinngemäß ,die Öffnung der Banken und Teile ihrer Daten für Drittanbieter“ (MoneyToday 2018). In einer weiterführenden Definition von McKinsey \& Company (2017) wird von einem kollaborativen Modell zum Austausch von Bankdaten durch APIs (Application Programming Interfaces) zwischen zwei oder mehreren selbstständigen Parteien gesprochen, welches das Ziel verfolgen, eine Funktionserweiterung für den Marktplatz zu schaffen. APIs sind Schnittstellen die es Entwicklern (Drittparteien) erlauben, ihre Applikation an die Software des Herausgebers der API anzubinden (MoneyToday 2018). APIs werden seit Jahrzehnten verwendet, insbesondere im Bereich der Finanzmanagementsoftware, bspw. um Rechnungsinformationen auf Bankwebseiten darzustellen oder um eine Verbindung zu Zahlungsnetzwerken wie Visa oder Master Card herzustellen. Die Schnittstellen dienten bis dato in erster Linie dem Austausch von Informationen und weniger dem Transfer von Geldern (PWC 2018, S. 3). Die innovative Nutzung von APIs ermöglicht Kunden nun aber neue und vielseitige Möglichkeiten im Bereich des Banking. Beispielsweise kann ein User Zahlungs- und Finanzapplikationen von Drittanbietern nutzen, welche mit Daten seiner bestehenden Bankverbindung(en) arbeiten. Die raschen technischen Fortschritte und die Vielfalt an Möglichkeiten erschweren die Eingrenzung von Anwendungsfeldern. Die Studie von PWC (2018) hat nach Analyse des Markts und nach Diskussionen mit Branchenvertretern folgende fünf Hauptkategorien von Anwendungsfeldern definiert: Aggregationsplattformen, Prozessoptimierungen, Beratungs- und Analysetools, erweiterte Produktangebote für Banken sowie erweiterte Nichtbanken-Produktangebote. Die Kategorienbildung wurde für die Einordnung der Anwendungsfälle in der vorliegenden Studie übernommen.

Ein wichtiger Aspekt bei der Identifizierung von Chancen und Risiken von Open Banking sowie bei der Analyse der Anwendungsfelder ist, wie in einem offenen System Geld verdient werden kann. Open Banking fokussiert insbesondere auf den Kundennutzen. Sind Kunden von der Customer Experience begeistert, wird angenommen, dass sie auch bereit sind, etwas dafür zu bezahlen. Die Wertschöpfung in Zusammenhang mit Open Banking kann in drei Gruppen differenziert werden: Erträge, Kosten und Unternehmenswert (PWC 2018, S. 15). Die Tab. 6.1 zeigt die drei Gruppen und mögliche Wertschöpfungsbereiche. 
Tab. 6.1 Wertschöpfungsmöglichkeiten im Kontext von Open Banking

\begin{tabular}{l|l|l|l|l}
\hline Erträge & $\begin{array}{l}\text { Pay for use: } \\
\text { Einmalige } \\
\text { Anfangsgebühr } \\
\text { oder abonnement- } \\
\text { basiertes Modell für } \\
\text { Dienstleistungen }\end{array}$ & $\begin{array}{l}\text { Provisionsmodell: } \\
\text { Provisionen für } \\
\text { empfohlene } \\
\text { Dienstleistungen } \\
\text { von Drittanbietern }\end{array}$ & $\begin{array}{l}\text { Werbung: } \\
\text { Mobile } \\
\text { Plattformen } \\
\text { nutzen, um } \\
\text { interne und } \\
\text { externe } \\
\text { Werbung zu } \\
\text { schalten }\end{array}$ & $\begin{array}{l}\text { Datenverkauf: } \\
\text { Verkauf von } \\
\text { gesammelten } \\
\text { Daten und } \\
\text { Trendanalysen }\end{array}$ \\
\hline Kosten & $\begin{array}{l}\text { Operationale Kosten: } \\
\text { Verwendung effizienter Dienstleistungen } \\
\text { von Drittanbietern, um die eigenen } \\
\text { operationellen Kosten zu senken. }\end{array}$ & $\begin{array}{l}\text { Investitionskosten: } \\
\text { Reduktion der Ausgaben durch } \\
\text { den Einsatz von Drittanbietern } \\
\text { bei der Entwicklung von } \\
\text { Anwendungen für die Kunden. }\end{array}$ \\
\hline Unternehmenswert & $\begin{array}{l}\text { Marktanteil: } \\
\text { Maximierung von Kundengewinnung und -bindung, um einen höheren } \\
\text { Unternehmenswert zu erreichen, indem die Produkte und Dienstleistungen } \\
\text { gratis oder zu stark reduzierten Preisen angeboten werden können. }\end{array}$ \\
\hline
\end{tabular}

Quelle: Eigene Darstellung in Anlehnung an PWC (2018)

Der Wandel der Rahmenbedingungen ist für Banken ein omnipräsentes Thema. Es wird versucht, sich möglichst optimal auf die veränderten Einflüsse einzustellen und für die Zukunft zu rüsten. Die aktuelle Situation ist aber grundlegend anders, denn der Veränderungsdruck ist zurzeit so intensiv und mit einer völlig neuen Geschwindigkeit, dass neue Wege gegangen werden müssen (Seidel 2016, S. 25-30). Die Auswirkungen auf die Geschäftsmodelle zeigen sich bereits und alle gängigen Modelle bedürfen einer kritischen Prüfung (Deloitte 2016, S. 1). In Europa müssen die Banken mit PSD2 per Gesetz Drittanbietern den Zugang zu spezifischen Kundendaten gewähren. In der Schweiz beruht dies auf freiwilliger Basis. Dennoch müssen die Banken und FinTech sich Gedanken über die Zusammenarbeit machen. In der Literatur finden sich unterschiedliche Definitionen von potenziellen Modellen zur Zusammenarbeit zwischen Banken und Drittanbietern, welche sich inhaltlich nicht stark differenzieren. MoneyToday (2019) spricht von einer Minimalund Maximalvariante und nennt Mischformen dazwischen. Die Studie von Innopay (2018) nennt fünf Stufen bis zur Maximalvariante des „Marketplace Banking“. Aus den genannten Modellen wird eine Reduktion auf drei unterschiedliche Zusammenarbeitsmodelle abgeleitet. Die Abb. 6.2 zeigt die drei Modelltypen, welche von Unternehmen adaptiert werden können: „,in-house model“, ,partnership model“ und „,market place model“. Beim „,in-house model“ bieten die Banken weiterhin die gesamten End-to-End Dienstleistungen im Hause an. Sie stellen sicher, dass ihr digitales Angebot konkurrenzfähig ist und versuchen organisch zu wachsen sowie Innovationen, welche von Kunden gewünscht werden, vom Markt zu kopieren. Anderseits werden auch Übernahmen von FinTechs angestrebt. Die Banken ermutigen ihre Kunden, auf den eigenen Plattformen zu bleiben und verfolgen generell eine defensive Strategie im Bereich der Öffnung von APIs (PWC 2018, S. 45). Beim ,partnership model“ suchen Banken spezifische Third Party Provider (TPP) aus, mit welchen sie zusammenarbeiten wollen. Unter Third Party Providern werden Drittanbieter 


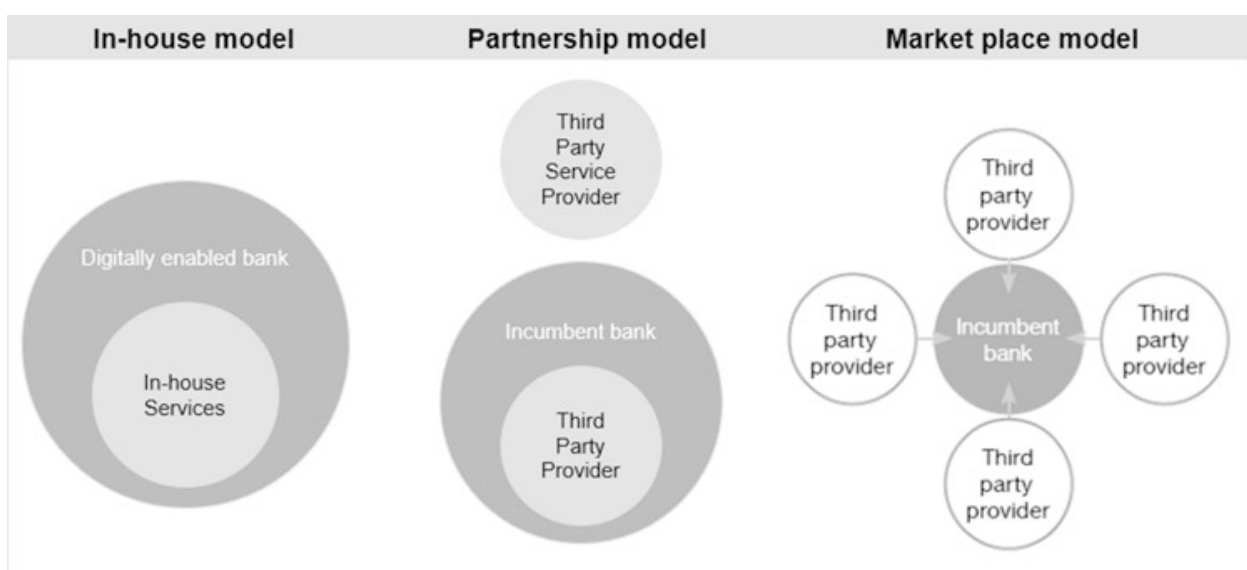

Abb. 6.2 Potenzielle Zusammenarbeitsmodelle. (Quelle: eigene Darstellung in Anlehnung an PWC 2018)

verstanden, welche über APIs Zugang zu Daten erhalten. Die Banken prüfen dabei, ob die potenziellen Partner vertrauenswürdig sind und einen Mehrwert für die Kunden bieten. Es ist vorstellbar, dass Exklusivitätsvereinbarungen unterzeichnet werden, um eine $\mathrm{Zu}$ sammenarbeit der TPPs mit direkten Konkurrenten zu verhindern. Die Banken würden Ökosysteme mit den Partnern errichten, um gemeinsame Lösungen für die Kunden anbieten zu können. Kunden werden dementsprechend ermutigt, die Dienstleistungen der anderen Mitglieder des Ökosystems zu nutzen. Durch die Partner kann das eigene Angebot optimal ergänzt und erweitert werden, es wird jedoch mit geschlossenen APIs gearbeitet (PWC 2018, S. 46). Beim „,market place model“ würden einige Unternehmen zu Hubs innerhalb des Systems werden. Das Modell fördert durch offenen APIs Innovationen durch Entwicklercommunities. Ein Beispiel dafür ist die API-Plattform der Deutschen Bank (dbAPI) (Deutsche Bank 2019). Die Partnerschaften sind bei diesem Modell nicht exklusiv und Kunden haben die Möglichkeit, von Funktionen und Dienstleistungen mehrerer Anbieter zu profitieren und haben eine aktive Rolle in der Auswahl von Produkten, Features und Dienstleistungen. Die Teilnehmer müssen sich dabei auf die Stärke ihrer Marke und auf ihre Wettbewerbsfähigkeit verlassen, um relevant zu bleiben (PWC 2018, S. 45).

\subsection{Forschungsfrage}

Die zentrale Forschungsfrage der vorliegenden Studie lautete:

\section{Welche Auswirkungen hat Open Banking auf die Schweizer Bankenindustrie?}

Die Untersuchung sollte aufzeigen, wie Open Banking die Finanzbranche transformieren kann und welche Folgen dies für Schweizer Banken hat. Um die zentrale Forschungs- 
frage beantworten zu können, wurden im Rahmen der Untersuchung drei Teilforschungsfragen definiert:

1. Wo steht die Schweiz im Kontext von Open Banking im internationalen Vergleich?

2. Welche Chancen und Risiken bietet der Ansatz und wie sehen mögliche Anwendungsfelder aus?

3. Wie verändern sich die Geschäftsmodelle der Schweizer Banken im Zuge von Open Banking und welche Handlungsoptionen lassen sich daraus ableiten?

Die erste Teilforschungsfrage öffnet das Forschungsfeld, indem der Stand von Open Banking im In- und Ausland untersucht wird. Aus dieser Analyse soll abgeleitet werden, welche Länder als Vorreiter gelten, um aus den dortigen Entwicklungen Schlussfolgerungen für den Schweizer Finanzplatz zu ziehen. Mittels der zweiten Teilforschungsfrage soll das Potenzial des Ansatzes eruiert werden. Dabei wird insbesondere wichtig sein, welche Anwendungsfälle für Schweizer Banken infrage kommen. Die dritte Forschungsfrage soll aufzeigen, wie sich das traditionelle Geschäftsmodell der Banken im Kontext von Open Banking verändern könnte und welche Handlungsoptionen Banken zur Wahl stehen.

Das Modell der Transformationstreiber der Bankenindustrie von Alt und Puschmann (2016) wird für die Darstellung der Forschungslücke adaptiert. Anhand der in Abb. 6.3 dargestellten drei Teilforschungsfragen kann der Untersuchungsbereich aufgezeigt werden.

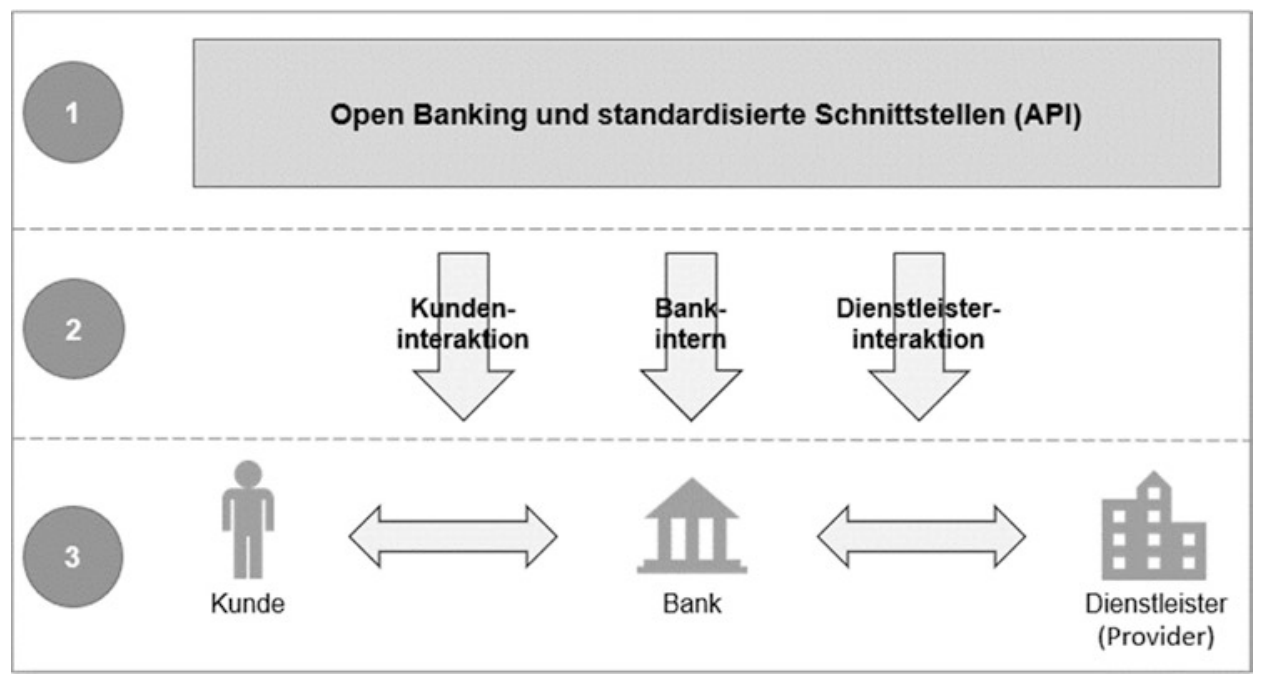

Abb. 6.3 Forschungslücke. (Quelle: eigene Darstellung) 


\subsection{Methodik}

Die Forschungsfrage wurde mithilfe von Experteninterviews bearbeitet. Der Einsatz eines Leitfadens wird in der Studie als strukturiertes Interview gewertet. Teilweise findet aber eine Verknüpfung mit der Technik des offenen Interviews statt, um gezielt Informationen und Erfahrungen der Experten abzuholen. Der Leitfaden für das Interview stellt das Instrument der Datenerhebung dar, ist aber gleichzeitig auch eine Übersetzung des Forschungsproblems und der theoretischen Annahmen in explizite Interviewfragen (Kaiser 2014, S. 52). Dabei wird auch von der Operationalisierung der Forschungsfrage gesprochen (Gläser und Laudel 2010, S. 132). Der thematische Aufbau richtet sich nach den Teilforschungsfragen. Es wurde auf eine nachvollziehbare Argumentationslogik geachtet, wobei von allgemeinen Aspekten auf spezielle Aspekte geschlossen wird (Kaiser 2014, S. 54).

Als Einführung wurde im Rahmen der Realisierung der Interviews die Forschungsarbeit und der Ablauf des Interviews erläutert sowie die organisatorischen Punkte (Aufnahme, Transkription, Anonymisierung) geklärt. Anschließend folgten zwei „warm-up“ Fragen, welche den Experten die Möglichkeit gaben, ins Gespräch einzufinden und eine entspannte Atmosphäre herzustellen (Przyborski und Wohlrab-Sahr 2013, S. 68). Als „,cool down“ wurde offen gefragt, ob die Experten noch weitere Inputs zum Thema haben und ob ihrer Meinung nach alle wichtigen Aspekte angesprochen wurden. Der Leitfaden für die Interviews wurde vor Durchführung einem Pretest unterzogen und anschließend anhand der Ergebnisse weiterentwickelt.

Die Auswahl der Experten war ein wesentlicher Aspekt des Forschungsdesigns. Als Experten wurden Personen gewählt, welche sich intensiv mit Open Banking befassen und in ihrem Unternehmen oder ihrer Institution entsprechend verantwortlich für die Thematik sind. Um eine Mehrdimensionalität zu erreichen, wurde außerdem Wert auf eine breite Verteilung aus unterschiedlichen Branchen gelegt bzw. auch innerhalb der Branche (z. B. differenzierende Bankgruppen).

Gesamthaft wurden neun Experteninterviews mit einer durchschnittlichen Dauer von 45-60 min im Zeitraum zwischen April und Mai 2019 durchgeführt. Bei den Experten handelte es sich um wichtige Entscheidungsträger innerhalb der Open-Banking-Bewegung der Schweiz, welche auch in nationalen Projekten wie Swiss Corporate API oder Common API mitwirken. Alle Interviews wurden in schriftdeutscher Sprache durchgeführt und transkribiert. Die Transkriptionen wurden anschließend mittels dem System Atlas.ti kodiert (Mayring und Fenzl 2014, S. 546). Die Kodierung richtet sich nach der Struktur des Leitfadens. In einer ersten Phase (,first-cycle-coding“) wurden den einzelnen Passagen Codes zugewiesen, welche dann zusammengefasst und reduziert wurden. In einer zweiten Phase (,second-cycle-coding“) wurden spezifischen Themen und Konzepten weitere Codes hinzugefügt. Dies ermöglichte eine systematische Strukturierung der Ergebnisse. Es wurden Codefamilien gebildet, denen die rund 40 verschiedenen Codes zugewiesen wurden. 


\subsection{Ergebnisse}

Im Nachfolgenden werden die Ergebnisse der empirischen Erhebung erläutert.

\subsubsection{Open Banking in der Schweiz und im internationalen Vergleich}

Die Resultate zeigen, dass die Präsenz von Open Banking in den letzten Jahren angestiegen ist und weiter zunehmen wird. Anhand der Einschätzung von Gartner Inc. (2018) im „Hype Cycle for Digital Banking Transformation“ soll Open Banking in 2-5 Jahren die Produktivitätsphase erreichen. Diese Wahrnehmung zeigte sich auch in den Experteninterviews. Die qualitative Erhebung veranschaulichte ebenfalls, dass nicht alle die gleiche Auffassung davon haben, wie Open Banking definiert wird. Im engeren Sinn bedeutet Open Banking die Öffnung der Banken mittels APIs gegenüber Drittanbietern. Im weiteren Sinne kann Open Banking als Bewegung in der Finanzwelt verstanden werden, die sich insbesondere mit der Zukunft des Banking befasst. In der Theorie wie auch in der qualitativen Erhebung zeigte sich, dass unabhängig davon, ob Open Banking als technische Entwicklung angesehen wird oder als größere Bewegung, es das Potenzial hat, die Bankenindustrie nachhaltig zu verändern. Der Veränderungsprozess wird aber eher als organisch betrachtet und weniger als rasche Disruption.

Die Resultate aus der Empirie bestätigten, dass das Vereinigte Königreich und deren Institute weltweit am meisten unternommen haben, um Open Banking voranzutreiben. Mit PSD2 holen nun die EU-Staaten auf. Insbesondere in Deutschland bieten die Banken bereits viele API-Funktionen an. Die Schweiz kann profitieren, indem sie die Entwicklungen in diesen Ländern verfolgt und ihre Schlüsse daraus zieht. Denn die Ergebnisse zeigen, dass Open Banking in der Schweiz noch in den „Kinderschuhen“ steckt. Eine äquivalente Regulierung zu PSD2 wird zwar von der Mehrheit nicht gewünscht, dennoch wird ihr eine stimulierende Wirkung für Open Banking Aktivitäten attestiert. Der Treiber Richtung Innovationen und Initiativen in diesem Bereich sollte der Kunde sein. Generell wird die Meinung vertreten, dass, wenn der Bedarf vom Kunden da ist, der Markt entsprechend reagieren wird. Die Banken treiben ihre Digitalisierungsstrategie hinsichtlich offener APIs weiter, um digitale Ökosysteme zu fördern. Zwei Projekte können hervorgehoben werden. Einerseits Corporate API von der SIX (neu: „SIX Connectivity Platform“), welches anhand der empirischen Ergebnisse vertieft betrachtet werden konnte. Das Projekt wird für die Schweizer Finanzindustrie von zentraler Bedeutung sein, denn es bietet die Möglichkeit, einen gemeinsamen Standard festzulegen und später die Anwendungsfälle zu erweitern. Anderseits „Finstar“, das Open-Banking-Ökosystem der Hypothekarbank Lenzburg. Die Lösung zeigt den Weg Richtung digitaler Ökosysteme und wie erfolgreich in einem Netzwerk mit unterschiedlichen Partnern gearbeitet werden kann. Bei den Schweizer Großbanken ist weiterhin unklar, ob sie die PSD2-Schnittstellen ihrer EU-Niederlassungen auch für Kunden in der Schweiz ausweiten werden oder ob sie an der Initiative der SIX festhalten werden. 


\subsubsection{Chancen und Risiken von Open Banking}

Die Ergebnisse zeigen, dass neue Open-Banking-Anwendungen schrittweise mit zunehmendem Kundennutzen und Kreativitätsniveau entwickelt werden. In der vorliegenden Studie wurden die Anwendungen in fünf Kategorien eingeteilt: Aggregationsplattformen, Prozessoptimierungen, Beratungs- und Analysetools, erweiterte Produktangebote für Banken und erweiterte Nichtbanken-Produktangebote. Es zeigt sich, dass Open Banking das Potenzial hat, auf alle Geschäftsbereiche der Banken einzuwirken und sowohl Privatals auch Geschäftskunden von neuen Möglichkeiten profitieren könnten. Aus der empirischen Erhebung lässt sich zudem interpretieren, dass die Unternehmen mit den ersten Use-Cases starten und anschließend anhand des Entwicklungsverlaufs weitere Anwendungen aufnehmen wollen. Die neuen Anwendungen zielen darauf hin, dem Kunden eine Vereinfachung der Prozesse zu ermöglichen. Daraus können neue Einnahmequellen und neue Geschäftsmodelle entstehen. Aus der Verknüpfung der Literaturanalyse mit den empirischen Ergebnissen lassen sich die Stärken und Schwächen von Banken sowie die Chancen und Risiken im Kontext von Open Banking ableiten. Die SWOT-Analyse in Abb. 6.4 dient als Übersicht für die nachfolgende Interpretation und als Grundlage für die Formulierung von Handlungsempfehlungen. Der Einsatz der SWOT-Analyse für die vorliegende Forschungsarbeit kann kritisch betrachtet werden, da sie als Werkzeug insbesondere für die Analyse interner Unternehmensfaktoren sowie externer Umweltfaktoren dient. Da die Institute im Bankensektor nicht homogen sind, wäre eine SWOT-Analyse grundsätzlich pro Bank zu erstellen. Im Kontext von Open Banking lassen sich aber gemeinsame Stärken und Schwächen der Banken ableiten. Demnach wird sie als geeignetes Analysetool für die Forschungsarbeit bewertet.

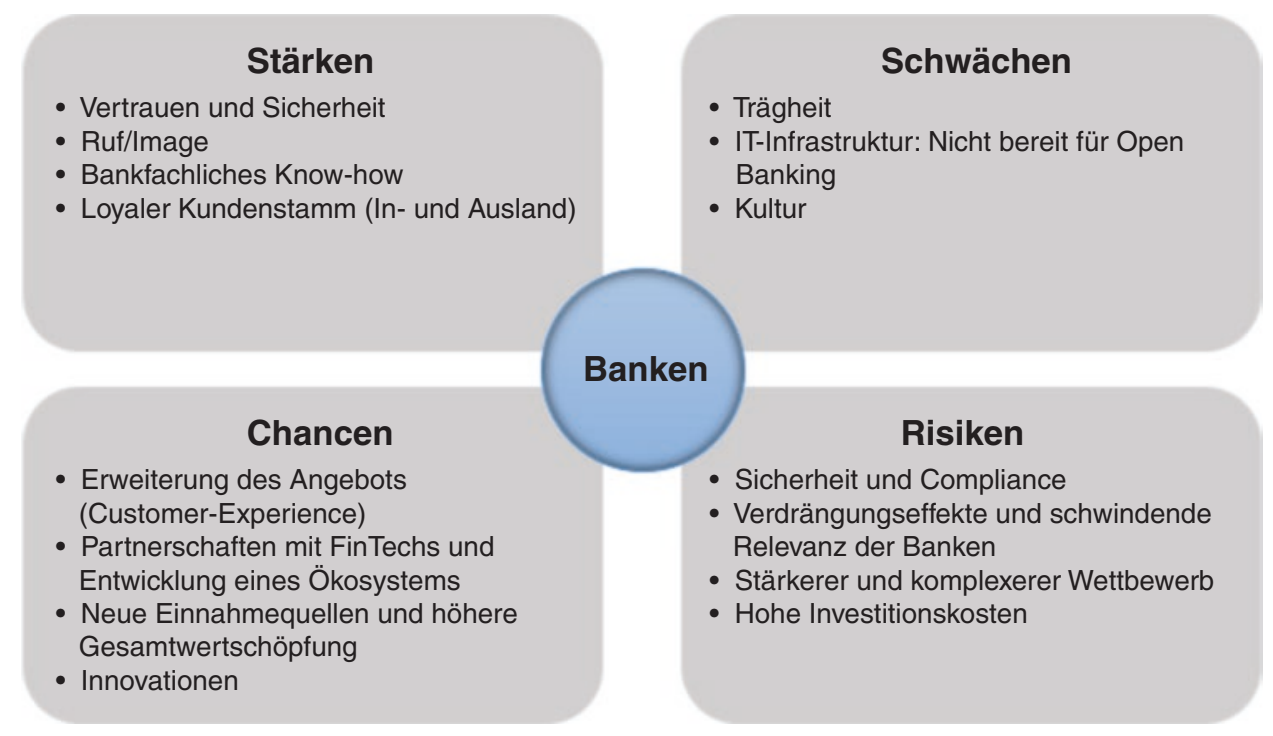

Abb. 6.4 SWOT-Analyse. (Quelle: eigene Darstellung) 
Die Ergebnisse zeigen, dass die etablierten Banken, verglichen mit anderen Marktteilnehmern wie Neobanken, FinTechs oder TPPs, immer noch über Stärken verfügen, um sich im Wettbewerb durchzusetzen. Sie verfügen aber auch über Schwächen, die für die Entfaltung des Potenzials von Open Banking hinderlich sind. Trotz der Bedrohungen lässt sich aus den Resultaten schließen, dass die Banken weiterhin stark positioniert sind, um sich den Herausforderungen zu stellen. Es scheint, als sind die Stärken und Schwächen der Banken spiegelverkehrt zu den Kompetenzen der anderen Marktteilnehmer zu sehen. Daraus lässt sich ableiten, dass eine Zusammenarbeit angestrebt werden sollte, um gemeinsam die Customer Experience zu verbessern.

\subsubsection{Einfluss auf die Geschäftsmodelle Schweizer Banken und strategische Handlungsoptionen}

Neben der Produktinnovationsperspektive eröffnet Open Banking auch im Bereich der Transformation von Geschäftsmodellen enormes Potenzial. Im Rahmen der Ergebnisse aus der qualitativen Erhebung und der Literaturanalyse, insbesondere unter Bezugnahme neuer Modelle, wurde der Wandel vom traditionellen, geschlossenen Geschäftsmodell zum offenen Geschäftsmodell betrachtet. Das entwickelte Modell in Abb. 6.5 orientiert sich an bereits existierenden Darstellungen.

Im traditionellen geschlossenen Geschäftsmodell werden den Kunden die Dienstleistungen im direkten Kanal von der Bank angeboten. Im offenen Geschäftsmodell werden die Kanäle erweitert und neue Lösungen von Drittanbietern integriert. Dabei entstehen

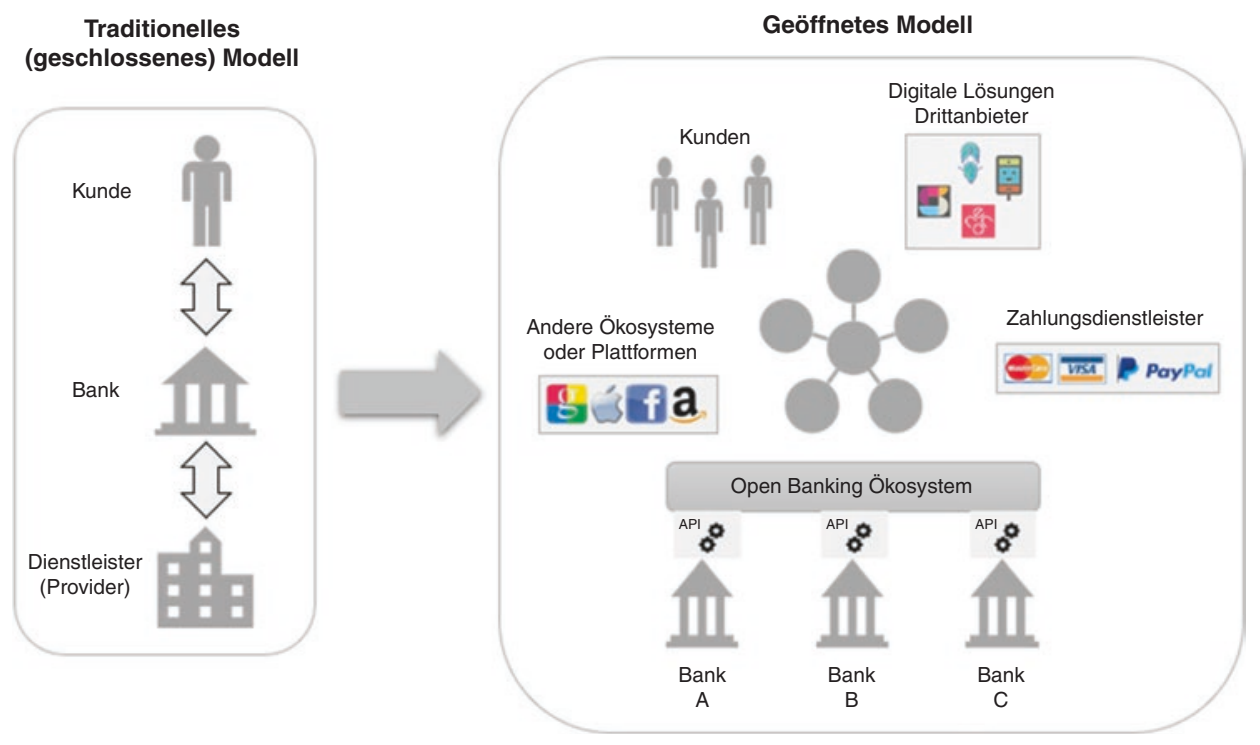

Abb. 6.5 Open-Banking-Geschäftsmodell. (Quelle: eigene Darstellung) 
neue Ökosysteme. Der Trend zu digitalen Ökosystemen wurde klar ersichtlich. Die Zusammenarbeit mit Partnern innerhalb des Ökosystems wird als essenziell beurteilt. Daraus lässt sich schließen, dass Banken, welche ein offenes Geschäftsmodell verfolgen, aktiv neue Partnerschaften suchen werden, um ihr Angebot zu erweitern und neue Einnahmequellen zu generieren. Im Zentrum des Netzwerks steht immer die Generierung eines Mehrwerts für den Kunden, aber auch für die anderen Teilnehmer. Es zeigt sich ebenfalls, dass unterschiedliche Visionen für digitale Ökosysteme in der Finanzwelt bestehen. Open Banking wird dabei als „Enabler“ solcher Systeme betrachtet. Die Wertschöpfungsmöglichkeiten im offenen Geschäftsmodell sind schwierig zu prognostizieren. Beim offenen Modell liegt der Fokus primär auf die Customer Experience und den Mehrwert für den Kunden. Die Einschätzungen ergaben aber, dass, wenn der Kunde von der Lösung begeistert ist, er auch bereit wäre, etwas dafür zu bezahlen.

Die Ergebnisse zeigen, dass offene Infrastrukturen die Banken in den nächsten Jahren beschäftigen werden. Dabei werden die Grenzen zwischen den Branchen weiter verwischen und die übergreifende Zusammenarbeit wird zunehmen. Weiter wird deutlich, dass nicht jede Bank die Ressourcen und Fähigkeiten für den Aufbau einer eigenen digitalen Plattform hat. Das bedeutet aber nicht, dass sie keine APIs und offenen Modelle verwenden sollten bzw. können, um Wertschöpfung zu generieren. Die qualitative Erhebung zeigte deutlich, dass alle Banken, unabhängig ihrer Größe oder Ausrichtung, eine Open-Banking-Strategie erarbeiten sollten. Unabhängig der definierten Strategie zeigten die Ergebnisse, dass es gemeinsame Erfolgsfaktoren für Banken der Zukunft gibt. Führende Banken der Zukunft werden demnach eine für den Kunden ganzheitliche, konsistente Customer Experience anbieten, die alles berücksichtigt, um ihn entlang des gesamten Prozesses zufriedenzustellen. Die Anbindung von Drittanbietern aus verschiedenen Sektoren wird wichtig sein, um überzeugende Lösungen anzubieten. Es lässt sich ableiten, dass für den Erfolg zudem der zielgerichtete Umgang mit Daten, der Aufbau einer agilen Organisation und die Schaffung von spannenden und sicheren Technologien entscheidend sein werden.

\subsection{Handlungsempfehlungen}

Die Ergebnisse dieser Forschungsarbeit zeigen, dass Banken, welche die Chancen von Open Banking nutzen wollen, die Auswirkungen und Implikationen auf die verschiedenen Dimensionen berücksichtigen müssen. Es zeigt sich, dass diese im Wesentlichen im Bereich der Strategie, Technologie (Datenmanagement), Compliance und im Aufbau eines Ökosystems liegen. Ziel sollte sein, das Geschäftsmodell so auszurichten, dass es für Drittanbieter geöffnet ist und den Kunden eine möglichst ideale „Ende-zu-Ende Customer Experience" bietet. Die Anbindung von weiteren Unternehmen wie FinTechs, Versicherungen, Zahlungsdienstleistern oder branchenfremden Unternehmen kann in diesem System einen Mehrwert bringen. Viele Finanzinstitute, insbesondere im Europäischen Raum, arbeiten seit Längerem im Rahmen von Open Banking mit APIs und nutzen Kooperationen. Services und Produkte, die über das klassische Banking hinausgehen, bieten fast 
unbegrenzte Möglichkeiten. Sie sind aber im Moment noch Visionen, die den Weg für die Zukunft aufzeigen können.

In der Schweiz hat sich gezeigt, dass viele Unternehmen sich rund um die Diskussionen von PSD2 und der Stellungnahme der Schweizerischen Bankiervereinigung SBVg noch bedeckt halten. Insbesondere die Banken beziehen meist eine abwartende Haltung und wollen aus den Erfahrungen der EU lernen. Dieser Ansatz ist sicherlich nicht falsch, birgt aber die Gefahr, zu viel Zeit zu verlieren, welche später schwer einzuholen ist. Die Ergebnisse der Forschungsarbeit zeigen, dass dem Konzept Open Banking großes Potenzial zugestanden wird und die Bedeutung in Zukunft zunehmen wird. Es wird jedoch von einem organischen Wachstum ausgegangen, wobei sich die Wünsche der Kunden und die Angebote der Unternehmen in Wechselwirkung gegenseitig beeinflussen. Es wird sich zeigen, wie hoch die Akzeptanz für neue Anwendungen ist. Auch wenn zu Beginn ein langsames Wachstum herrschen könnte, dürfte sich Open Banking zu gegebener Zeit exponentiell ausbreiten. Für die Banken ist demnach wichtig, sich rechtzeitig zu positionieren und ihr Geschäftsmodell, ihre IT und die Prozesse anzupassen. Diese Entwicklung benötigt viel Zeit und Ressourcen, die dann möglicherweise nicht (mehr) zur Verfügung stehen. Die erwähnten Aspekte führen zum Ergebnis, dass die Schweizer Banken auch ohne gesetzlichen Druck eine der vier Umsetzungsstrategien für Open Banking (Comply, Growth, Competition und Change), welche in der Abb. 6.6 visualisiert sind, definieren sollten. Denn unabhängig vom potenziellen Umfang oder Zeitrahmen von Open Banking hilft eine klare Strategie als Entscheidungshilfe, welche Fähigkeiten aufgebaut werden müssen, um im Wettbewerb weiterhin bestehen zu können. Dabei können sie sich auch auf ihre Stärken besinnen, welche im Rahmen dieser Forschung erkannt wurden. Wünschbar

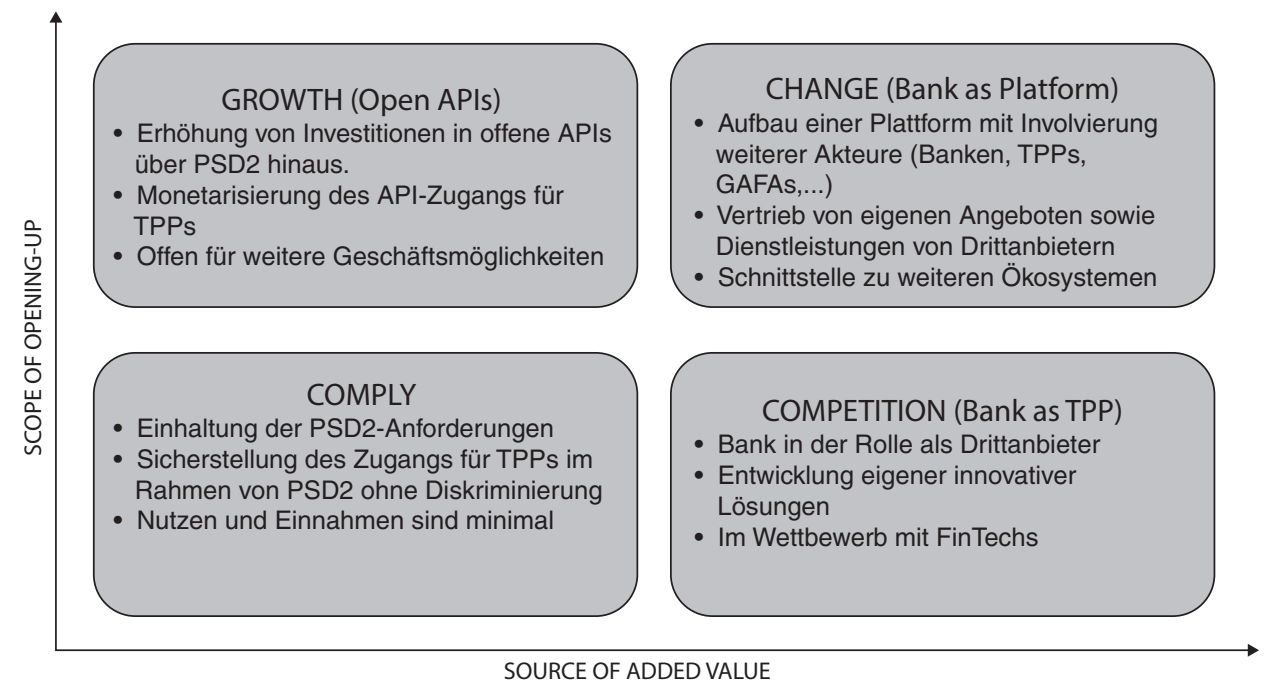

Abb. 6.6 Umsetzungsstrategien Open Banking. (Quelle: eigene Darstellung in Anlehnung an BEI 2017) 
für die Schweiz und ihre Institute bleibt, dass sie die Entwicklungen nicht verschlafen und mit Innovationen in der ersten Reihe mitspielen können.

\section{$6.7 \quad$ Fazit}

In der Ausgangslage dieser Forschungsarbeit wurde Open Banking als signifikanter Trend im Bereich der Finanzindustrie beschrieben und gleichzeitig als Möglichkeit für die Banken, sich dem herausfordernden Marktumfeld zu stellen. Die Ergebnisse unterstreichen die zukünftige Bedeutung von Open Banking für die Schweizer Bankenindustrie und anhand der Beantwortung der Teilforschungsfragen lassen sich die Auswirkungen ableiten. Der aktuelle Stand von Open Banking in der Schweiz zeigt, dass die Entwicklungen im Vereinigten Königreich, aber auch in vielen EU-Staaten als Maßstab verwendet werden können. Für eine einheitliche Beurteilung im Sinne eines Reifegrades wäre jedoch die Entwicklung eines Modells wünschenswert. Die vorliegende Forschung liefert hier keine abschließenden Ergebnisse. Die Resultate zeigen aber die Bemühungen von Schweizer Instituten, einen einheitlichen Standard zu implementieren und Open Banking voranzutreiben. Als Grundlage für strategische Überlegungen konnte anhand der Erkenntnisse aus der Literaturrecherche und der Experteninterviews eine SWOT-Analyse für Schweizer Banken im Kontext von Open Banking erarbeitet werden. Sie verdeutlicht, dass sich Banken auf ihre Stärken wie ihr Image als sicherer und vertrauenswürdiger Partner besinnen sollten, um gegen neue Wettwerber im Markt zu bestehen. Die Chancen liegen außerdem bei der Verbesserung der Kundenerlebnisse mit der Erweiterung von Produkten und Services sowie dem Aufbau eines Ökosystems. Es zeigte sich, dass Experten die Chancen gegenüber den Risiken höher gewichten. Die Zukunft wird zeigen, welche Akteure effektiv die Vorteile von Open Banking nutzen werden, um ihre Position im umkämpften Markt zu stärken. Die Transformation der Geschäftsmodelle und der IT werden dabei einen wichtigen Faktor darstellen. Grundsätzlich ließ sich aus den Ergebnissen feststellen, dass sich die Geschäftsmodelle öffnen werden und multilaterale Beziehungen zunehmen werden. Die Frage, wie die Branche in fünf Jahren aussieht, bleibt offen. Digitale Ökosysteme werden sicherlich an Relevanz gewinnen. Wie die Wertschöpfung darin aussehen wird, konnte aber ebenfalls nicht abschließend geklärt werden. Die Forschungsarbeit liefert aber, begünstigt durch das spezifische Wissen der Experten, wichtige Erkenntnisse und Handlungsempfehlungen für die Praxis und die weitere Forschung.

\section{Literatur}

Accenture. (2018). The brave new world of open banking. https://www.accenture.com/_acnmedia/ PDF-77/Accenture-Brave-New-World-Open-Banking.pdf. Zugegriffen am 27.12.2018.

Alt, R., \& Puschmann, T. (2016). Digitalisierung der Finanzindustrie: Grundlagen der FintechEvolution. Berlin: Springer Gabler. 
BEI, Christian WilhelmChristian Wilhelm war bis 2018 Mitarbeiter des. (2017). PSD2 - Auf dem Weg zum Open Banking. ccecosystems.news.

Deloitte. (2016). Zukünftige Geschäftsmodelle für Schweizer Banken. https://www2.deloitte.com/ $\mathrm{ch} / \mathrm{de} /$ pages/financial-services/articles/swiss-banking-business-models-of-the-future.html. $\mathrm{Zu}$ gegriffen am 27.12.2018.

Deutsche Bank. (2019). Developer portal. https://developer.db.com/. Zugegriffen am 24.04.2019.

Fasel, D., \& Meier, A. (2016). Big Data: Grundlagen, Systeme und Nutzungspotenziale. Wiesbaden: Springer Vieweg.

Fitzgerald, M., Kruschwitz, N., \& Welch, M. (2013). Embracing digital technology: A new strategic imperative (case study). https://sloanreview.mit.edu/projects/embracing-digital-technology/. Zugegriffen am 27.12.2018.

Gartner Inc. (2018). Hype cycle research methodology. Gartner Inc. https://www.gartner.com/technology/research/methodologies/hype-cycle.jsp. Zugegriffen am 07.06.2018.

Gläser, J., \& Laudel, G. (2010). Experteninterviews und qualitative Inhaltsanalyse: als Instrumente rekonstruierender Untersuchungen (4. Aufl.). Wiesbaden: VS Verlag für Sozialwissenschaften.

Innopay. (2018). Mastering open banking: How the masters in openness create value. https://www. innopay.com/en/publications/mastering-open-banking-how-masters-openness-create-value. Zugeriffen am 04.01.2018.

Kaiser, R. (2014). Qualitative Experteninterviews: Konzeptionelle Grundlagen und praktische Durchführung. Wiesbaden: VS Verlag für Sozialwissenschaften (Elemente der Politik).

Matt, C., Hess, T., \& Benlian, A. (2015). Digital Transformation Strategies. Springer. https://doi. org/10.1007/s12599-015-0401-5.

Mayring, P., \& Fenzl, T. (2014). Qualitative Inhaltsanalyse. In N. Baur \& J. Blasius (Hrsg.), Handbuch Methoden der empirischen Sozialforschung (S. 543-556). Wiesbaden: Springer Fachmedien. https://doi.org/10.1007/978-3-531-18939-0_38.

McKinsey \& Company. (2017). Data sharing and open banking. https://www.mckinsey.com/ /media/McKinsey/Industries/Financial\%20Services/Our\%20Insights/Data\%20sharing\%20and\%20 open\%20banking/Data-sharing-and-open-banking.ashx. Zugegriffen am 27.12.2018.

MoneyToday. (2018). Ist Open Banking der neue Wettbewerbsfaktor für Banken? MoneyToday. https://www.moneytoday.ch/news/ist-open-banking-der-neue-wettbewerbsfaktor-fuer-banken/. Zugegriffen am 22.12.2018.

MoneyToday. (2019). PSD2. MoneyToday. https://www.moneytoday.ch/lexikon/psd2/. Zugegriffen am 11.04.2019.

Nora, S., \& Minc, A. (1979). Die Informatisierung der Gesellschaft. Frankfurt a. M.: Campus.

NZZ. (2018). Auf der Bremse beim Open Banking? NZZ. https://www.nzz.ch/finanzen/auf-derbremse-beim-open-banking-ld.1392394. Zugegriffen am 22.12.2018.

Ornau, F. (2017). Digitalisierung in Wirtschaft und Wissenschaft. Wiesbaden: Springer (Weiterbildung und Forschung der SRH Fernhochschule - The Mobile University).

Peter, M. K. (2017). KMU-Transformation: Als KMU die Digitale Transformation erfolgreich umsetzen.: Forschungsresultate und Praxisleitfaden. Olten: FHNW Fachhochschule Nordwestschweiz.

Pousttchi, K. (2017). Digitale Transformation - Enzyklopädie der Wirtschaftsinformatik. http:// www.enzyklopaedie-der-wirtschaftsinformatik.de/lexikon/technologien-methoden/Informatik\%2D\%2DGrundlagen/digitalisierung/digitale-transformation/digitale-transformation/. Zugegriffen am 22.12.2018.

Przyborski, A., \& Wohlrab-Sahr, M. (2013). Qualitative Sozialforschung: Ein Arbeitsbuch. München: de Gruyter.

PWC. (2018). The future of banking is open. https://www.pwc.co.uk/industries/financial-services/ insights/seize-open-banking-opportunity.html. Zugegriffen am 27.12.2018. 
Schweizerische Bankiervereinigung. (2017). Positionspapier der SBVg. https://www.swissbanking. org/de/themen/archiv/aktuell/20170707-5000-all-positionspapier-psd2.pdf. Zugegriffen am 27.12.2018.

Seidel, M. (2016). Banking \& Innovation 2016: Ideen und Erfolgskonzepte von Experten für die Praxis. Berlin: Springer.

Wade, M. R. (2015). Digital business transformation. IMD Business School. https://www.imd.org/ contentassets/d0a4d992d38a41ff85de509156475caa/framework. Zugegriffen am 19.05.2019.

Marco Birkhofer Abgeschlossenes Masterstudium in Betriebswirtschaft an der Berner Fachhochschule mit Vertiefung Corporate and Business Development und davor Bachelorstudium in Business Administration mit Vertiefung Strategy, ebenfalls an der Berner Fachhochschule. Derzeit als Segmentsmanager Privatkunden bei der Valiant Bank AG tätig und dabei insbesondere verantwortlich für die strategische Weiterentwicklung des Segments sowie der Value Proposition gegenüber dem Kunden.

Sandro Bächli Professor für Banking \& Finance und Leiter der Fachgruppe Accounting, Finance, Tax der Abteilung Methoden und Grundlagen am Departement Wirtschaft der Berner Fachhochschule. Lehr- und Forschungstätigkeit in den Bereichen Investitionsrechnung und Unternehmensbewertung, Finanzierung und Finanzmärkte, Asset Allokation und Portfoliomanagement. Bankerfahrung als Investmentstratege.

Open Access Dieses Kapitel wird unter der Creative Commons Namensnennung 4.0 International Lizenz (http://creativecommons.org/licenses/by/4.0/deed.de) veröffentlicht, welche die Nutzung, Vervielfältigung, Bearbeitung, Verbreitung und Wiedergabe in jeglichem Medium und Format erlaubt, sofern Sie den/die ursprünglichen Autor(en) und die Quelle ordnungsgemäß nennen, einen Link zur Creative Commons Lizenz beifügen und angeben, ob Änderungen vorgenommen wurden.

Die in diesem Kapitel enthaltenen Bilder und sonstiges Drittmaterial unterliegen ebenfalls der genannten Creative Commons Lizenz, sofern sich aus der Abbildungslegende nichts anderes ergibt. Sofern das betreffende Material nicht unter der genannten Creative Commons Lizenz steht und die betreffende Handlung nicht nach gesetzlichen Vorschriften erlaubt ist, ist für die oben aufgeführten Weiterverwendungen des Materials die Einwilligung des jeweiligen Rechteinhabers einzuholen.

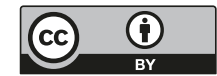

